\title{
Corrigendum: 2018 Korean Society for the Study of Obesity Guideline for the Management of Obesity in Korea
}

Mi Hae Seo' ${ }^{1}$ Won-Young Lee ${ }^{2}$, Sung Soo Kim ${ }^{3, *}$, Jae-Heon Kang ${ }^{4}$, Jee-Hyun Kang ${ }^{5}$, Kyoung Kon Kim ${ }^{6}$, Bo-Yeon Kim ${ }^{7}$, Yang-Hyun Kim ${ }^{8}$, Won-Jun Kim ${ }^{9}$, Eun Mi Kim ${ }^{10}$, Hyun Soo Kim ${ }^{11}$, Yun-A Shin ${ }^{12}$, Hye-Jung Shin ${ }^{13}$, Kyu Rae Lee ${ }^{14}$, Ki Young Lee ${ }^{15}$, Sang Yeoup Lee ${ }^{16}$, Seong-Kyu Lee ${ }^{17}$, Joo Ho Lee ${ }^{18}$, Chang Beom Lee ${ }^{19}$, Sochung Chung ${ }^{20}$, Young Hye Cho ${ }^{21}$, Kyung Mook Choi ${ }^{22}$, Jung Soon $\mathrm{Han}^{23}$, Soon Jib Yoo ${ }^{24, *}$; Committee of Clinical Practice Guidelines, Korean Society for the Study of Obesity (KSSO) 'Division of Endocrinology, Department of Internal Medicine, Soonchunhyang University Gumi Hospital, Soonchunhyang University School of Medicine, Gumi; 2Division of Endocrinology and Metabolism, Department of Internal Medicine, Kangbuk Samsung Hospital, Sungkyunkwan University School of Medicine, Seoul; ${ }^{3}$ Department of Family Medicine, Chungnam National University Hospital, Chungnam National University College of Medicine, Daejeon; ${ }^{4}$ Department of Family Medicine, Kangbuk Samsung Hospital, Sungkyunkwan University School of Medicine, Seoul; ${ }^{5}$ Department of Family Medicine, Konyang University Hospital, Konyang University College of Medicine, Daejeon; ${ }^{6}$ Department of Family Medicine, Gil Medical Center, Gachon University College of Medicine, Incheon; ${ }^{7}$ Division of Endocrinology and Metabolism, Department of Internal Medicine, Soonchunhyang University Bucheon Hospital, Soonchunhyang University College of Medicine, Bucheon; ${ }^{8}$ Department of Family Medicine, Korea University College of Medicine, Seoul; ${ }^{9}$ Department of Endocrinology and Metabolism, Gangneung Asan Hospital, University of Ulsan College of Medicine, Gangneung; ${ }^{10}$ Department of Dietetics, Kangbuk Samsung Hospital, Seoul; "Department of Sport Science, Seoul National University of Science and Technology, Seoul; ${ }^{12}$ Department of Exercise Prescription and Rehabilitation, Dankook University, Cheonan; ${ }^{13}$ Department of Pediatrics, National Medical Center, Seoul; ${ }^{14}$ Department of Family Medicine, Dongincheon Gil Hospital, Gachon University of Medicine and Science, Incheon; ${ }^{15}$ Division of Endocrinology and Metabolism, Department of Internal Medicine, Gachon University Gil Medical Center, Incheon; ${ }^{16}$ Department of Family Medicine, Pusan National University Yangsan Hospital, Pusan National University School of Medicine, Yangsan; ${ }^{17}$ Department of Internal Medicine, Eulji University Hospital, Eulji University School of Medicine, Daejeon; ${ }^{18}$ Department of Surgery, Ewha Medical Center, Seoul; ${ }^{19}$ Division of Endocrinology and Metabolism, Department of Internal Medicine, Hanyang University Guri Hospital, Hanyang University College of Medicine, Guri; ${ }^{20}$ Department of Pediatrics, Konkuk University Medical Center, Konkuk University School of Medicine, Seoul; ${ }^{21}$ Family Medicine Clinic and Research Institute of Convergence of Biomedical Science and Technology, Pusan National University Yangsan Hospital, Yangsan; ${ }^{22}$ Division of Endocrinology and Metabolism, Department of Internal Medicine, Korea University Guro Hospital, Korea University College of Medicine, Seoul; ${ }^{23}$ Research Institute of Human Ecology, Korea University, Seouli ${ }^{24}$ Division of Endocrinology and Metabolism, Department of Internal Medicine, Bucheon St. Mary's Hospital, College of Medicine, The Catholic University of Korea, Bucheon, Korea

J Obes Metab Syndr 2019;28:40-45

https://doi.org/10.7570/jomes.2019.28.1.40

We have noticed an error in Table 1 in our published paper above. Without abdominal obesity, obesity class I, II and III carry respective risks of comorbidities that are moderate, severe, and very severe. The authors sincerely apologize for this error and the revised table is shown below.
Table 1. Risk of comorbidity according to obesity and abdominal obesity ${ }^{12}$

\begin{tabular}{lccc}
\hline \multirow{2}{*}{$\begin{array}{l}\text { Classification } \\
\text { Sody mass index } \\
\left(\mathrm{kg} / \mathrm{m}^{2}\right)\end{array}$} & \multicolumn{2}{c}{$\begin{array}{r}\text { Risk of comorbidity according to } \\
\text { abdominal obesity }\end{array}$} \\
\cline { 3 - 4 } & & $\begin{array}{c}<90 \mathrm{~cm} \text { (men), } \\
<85 \mathrm{~cm} \text { (women) }\end{array}$ & $\begin{array}{c}\geq 90 \mathrm{~cm} \text { (men), } \\
\geq 85 \mathrm{~cm} \text { (women) }\end{array}$ \\
\hline Underweight & $18.5-22.9$ & Low & Average \\
Normal & $23-24.9$ & Average & Increased \\
Pre-obese & $25-29.9$ & Moderate & Moderate \\
Obese class I & $30-34.9$ & Severe & Vevere \\
Obese class II & $\geq 35$ & Very severe & Very severe \\
\hline Obese class III & &
\end{tabular}

Pre-obese may be defined as overweight or at-risk weight, and obese class III may be defined as extreme obesity. 